\title{
Laboratory Construction and Management of University based on Cloud Computing
}

\author{
Quan Jin Juan, Yang Jie \\ Tianjin University of Finance and Economics, Tianjin, China, 300222 \\ Email: qjj_16@163.com
}

\section{Keywords: Cloud Computing, Virtual Technology, Laboratory Construction}

\begin{abstract}
At present, the laboratories of universities are facing problems of high-cost, low utilization and hard to manage. Cloud computing can use several workstations and terminal clients to form a virtual computing lab and build a lab workstation platform through cloud computing architecture. This paper proposes a solution about university laboratory construction based on cloud computing to overcome the difficulties in traditional computer laboratory construction. It can effectively reduce the allocation cost, enhance management efficiency and strengthen data security.
\end{abstract}

\section{Introduction}

Experimental teaching plays an increasingly significant role in higher education. Computer laboratory in universities is often an important index to measure the quality of running a school. At present, many laboratories in universities are facing equipment aging and funds are limited. It needs a large amount of human resources, material resources and financial resources to update. Higher education must consider new technology to solve the above problems [1, 2].

Cloud computing has recently emerged as a new paradigm for hosting and delivering services over internet [8]. It provides services via virtual resources and internet. Cloud computing can assign resources dynamically according to the needs of users. It is argued that the Cloud computing has a significant place in the higher education landscape both as a ubiquitous computing tool and a powerful platform that can enhance engagement among educational researchers to understand and improve practice, and thus, increase the quality of their students' learning outcomes $[1,4]$.

This paper proposes an experimental environment using cloud computing technology. It can assign resources dynamically, reduce the cost, make the system easy to maintain and without geographical restrictions.

\section{The Concept and Characteristics of Cloud Computing}

1.1 What is Cloud Computing? Cloud computing is a term for networked computers that deliver IT services over the internet to many users in an on-demand environment. The types of services range from adaptations of familiar tools to address customers' various needs, ranging from scientific research to e-commerce [4]. It is a product of the development of computer and traditional network technology, such as Grid Computing, Parallel Computing, Distributed Computing and Utility Computing. There are three major types of cloud computing services [5]. They are:

(1) Infrastructure-as-a-service: this type of cloud provides virtual hardware capacity to organizations on an elastic basis. It packages the CPU, memory and network into service provided to the client. The client does not manage the infrastructure of computer and only operate the system and running a variety of applications. Amazon was the pioneer in this providing virtual machine instances, storage, and computation as innovative services in this category.

(2) Platform-as-a-service: Pass is higher than Iass, it provides a platform for cloud computing application programs. In this service model, the clients can create, test and manage their applications and service. But the clients can't control the infrastructure.

(3)Software-as-a-service: this type of cloud provides a wide range of applications (software) tools to end users. Any web application is a cloud application in the sense that it resides in the cloud. The users need not install software on their host and to use them by internet. Google Docs (for word 
processing and spreadsheets), YouTube, SlideShare, Amazon, Facebook, twitter, flickr, and virtually every other Web 2.0 application is a cloud application in this sense [4].

1.2 Virtual Technology. Cloud computing realize a verity of application through virtual technology. The virtual technology is to convert a physical unit into several virtual logic units, which can be used by multiple applications. The virtual entities are all kinds of IT resources, including memory virtualization, storage virtualization, hardware virtualization, software virtualization and system virtualization, etc. The virtual technology breaks the isolation between the hardware. All of the equipment in the network is managed uniformly and formed a shared resource pool. The resource is managed as an entity by the management program and the hardware resource is reconfigured dynamically according to the different load in order to achieve resource sharing, resource customization and on-demand service [6].

1.3 The Characteristics of Cloud Computing. Cloud computing is a dynamic scalable calculation. It provides service by virtual resource and internet. The user need not know the technical architecture of it. Cloud computing have bring a new revolution to the traditional calculation method. It has flexible scalability and can allocate resource dynamically according to user needs. It has improved the efficiency of resource use that the application software is no longer installed on the local computer and reduced the cost of hardware. The characteristic of cloud computing brings new opportunities for experiment teaching environment [3].

\section{The Advantages of the Application of Cloud Computing Technology in the Computer Laboratory in University}

On a research about the cloud computing application in teaching, Armando Fox [7] think it is feasible to apply cloud computing in teaching. Students can know about many new technology and tools about cloud computing through the teaching activities. Cloud computing can meet the students' requirements of using data server. The advantages of the application of cloud computing in teaching in lab are as follows:

(1)Reduce laboratory investment and improve the utilization rate of equipment. Cloud computing does not require high configuration of terminal computer, so the lab of cloud computing can use the computers that will be eliminated. This can avoid to update equipment frequently and to reduce the lab construction cost. At the same time, it only needs to install a set of application software on the server and saves the software purchase cost.

(2)Make laboratory management and maintenance convenient. The application of cloud computing technology can manage the software and hardware resources uniformly. It reduces the management workload to update and maintain the system. The manager only operates on the server and it reduces the cost and improves the efficiency of management.

(3)Break through the limit of time and place for laboratory use. Cloud computing can provide various, multi-level resources sharing service to users, such as $\mathrm{P} 2 \mathrm{C}, \mathrm{C} 2 \mathrm{C}$ and $\mathrm{P} 2 \mathrm{P}$. It is different from the traditional platform that many universities have at present. The Cloud computing provides more perfect teaching resource sharing service via public cloud service platform. Students and teachers can study whenever and wherever possible.

\section{Construction of the Experimental Platform based on Cloud Computing}

As shown in figure 1, the overall architecture of cloud computing lab is divided into 4 layers. They are cloud infrastructure hardware layer, virtual resource manage layer, virtual desktop manage layer and cloud terminal access layer [8]. 


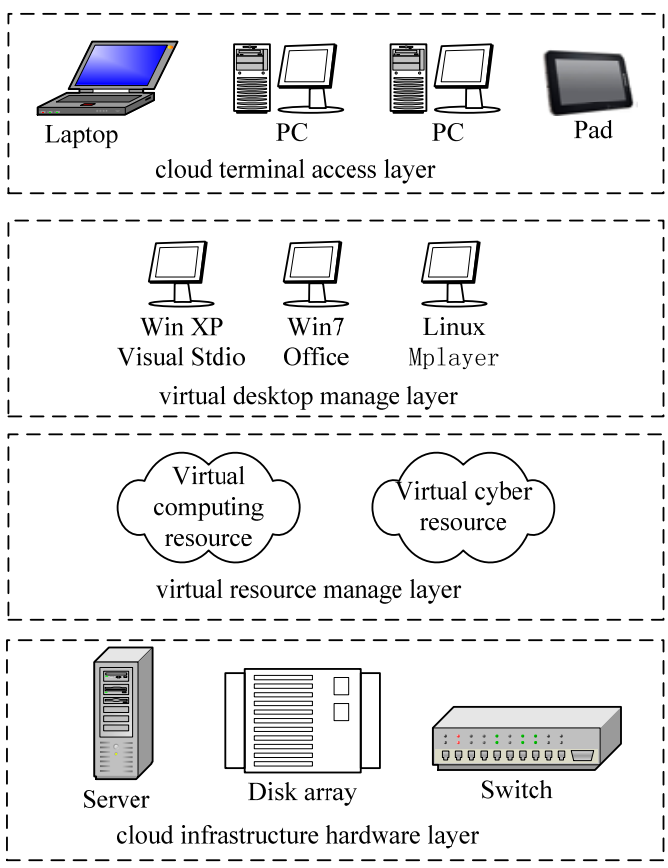

Figure 1. Construction of the Experimental Platform

(1)Cloud Infrastructure Hardware Layer. This layer includes computing equipment, network switching equipment and data storage device. They are connected with each other through network.

(2)Virtual Resource Manage Layer. This layer virtualizes the IT resource and transforms the physical resources into logic resource. It shields the complexity of physical resources and presents simple logic form. Therefore the complexity of management is reduced and the utilization rate of resources is improved. It realizes the unified management of computing resources, storage resources and network resource.

(3)Virtual Desktop Manage Layer. This layer is responsible for the entire virtual desktop system scheduling. It allocates virtual desktop system requests of different users. The users are authorized to use the desktop through identity certification with control system. They can manage the experimental data, user data, resource data and system data.

(4)Cloud Terminal Access Layer. The cloud terminal provides a standardized interface to user and cloud computing applications. In this paper, the cloud client programs are installed on the terminal computers of lab. The terminal computer login the cloud server to acquire high performance software service and mass storage service and etc. For example, when the students want run some software installed on the cloud server, such as Microsoft Office, Visual studio, Photo Shop, SQL server and so no, they can use them just after login.

\section{The Application of the Experiment Platform based on Cloud Computing}

On the basis of the above design scheme, without changing the existing network structure, by combining the virtualization technology and cloud computing, the computer laboratory constructs virtual machine cluster in the Intranet [9]. The specific implementation steps are as follows:

(1)Install Windows 2008(64bit), DNS, VMware vCenter Server and related plug-in service on a high performance server.

(2)Select a general computer to install vSphere Client, it provides the main connection way as interaction management with VMware vSphere system. Install vCenter Client on this computer to manage. The vSphere Client can be used as management interface and operation control console of every virtual machine of vCenter Server so as to provide available services to the users.

(3)Database is used to lay out the data in VMware vSphere environment. The vCenter Server can support many different databases, such as Microsoft SQL Server 2008 Express, oracle and etc.

(4)For each computer in the lab, specify IP address or virtual machine host name, input the user name and password that the administrator assigned. Thus, the user can access each virtual machine in the cloud platform directly. They can also open the vSphere Virtual machine management 
interface in the browser to create virtual machine and install the software needed.

\section{Summary}

This paper presents lab construction project based on Cloud computing. The Installation, updating and maintenance of software are all operated in vCenter Server. The cloud platform provides different services to meet the requirements of different teaching activities. Students can use any operating system they needed, such as Windows XP, Window 2003、Windows7,Liux and Unix. It reduces lab investment and improves the utilization rate of equipment. At the same time, it makes the management and maintenance of software convenient. The users can access the system whenever and wherever possible. The construction project worth being further popularized to provide more convenient experimental environment to students and teachers in university.

\section{References}

[1] Nan Ma, Mei Yuan, Guo liang Cao. Integration of Digital Campus Resources Based on Cloud Computing[C]. Proceedings of the 2012 International Conference on Cybernetics and Informatics Lecture Notes in Electrical Engineering Vol.163 (2014), p. 1957-1963

[2] Kong Yi.Uses of Cloud Computing in Laboratory Construction and Management of University and Colleges[J].China Education Technoloay \&Equipment.Vol.12(2013),p.28-31

[3] Sadiku, M.N.O, Musa, S.M., Momoh, O.D. Cloud Computing: Opportunities and Challenges Potentials, IEEE Vol.33 (2014), p.34 - 36

[4] P.Y.Thomas. Cloud computing.A potential paradigm for practising the scholarship of teaching and learning[J] ,The Electronic Library vol.29(2011),p.214-224

[5] Zhang Q., Cheng L., Boutaba R. Cloud computing: state-of-the-art and research challenges[J]. Journal of Internet Services and Applications vol.1(2010),p.7-18

[6] Stanoevska-Slabeva K, Wozniak T. Cloud Basics-An Introduction to Cloud Computing[J].Grid and Cloud Computing vol.1(2010),p.47-61

[7]Armando Fox.Cloud computing in education[EB/OL].http://inews.berkeley. edu/articles/Spring2009/cloud computing

[8] Singh MA, Shrivastava M. Overview of Security issues in Cloud Computing [J]. International Journal of Advanced Computer Research vol.2 (2012)p.41-45.

[9] ZHANG chen-xiang, ZHU Dan-chen:Construction Plan Optimization for College Computer Laboratories Based on Cloud Computing[J].Journal of Huaiyin Institute of Technology Vol.22(2013),p.28-30 